\title{
The Summer School Alpbach
}

\author{
Michaela Gitsch $^{1, *}$ and Periasamy K Manoharan ${ }^{2}$ \\ 1 Summer School Director, Austrian Research Promotion Agency (FFG), Sensengasse 1, 1090 Wien, Austria \\ 2 Radio Astronomy Centre, National Centre for Radio Astrophysics, Tata Institute of Fundamental Research, \\ 643001 Udhagamandalam (Ooty), India \\ *Corresponding author: michaela.gitsch@ffg.at
}

Received 28 December 2014 / Accepted 16 January 2015

\begin{abstract}
Sixty young, highly qualified European science and engineering students converge annually for stimulating 10 days of work in the Austrian Alps. Four teams are formed, each of which designs a space mission, which are then judged by a jury of experts. Students learn how to approach the design of a satellite mission and explore new and startling ideas supported by experts. The Summer School Alpbach enjoys more than 30 years of tradition in providing in-depth teaching on different topics of space science and space technology, featuring lectures and concentrated working sessions on mission studies in self-organised working groups. The Summer School is organised by the Austrian Research Promotion Agency (FFG) and co-sponsored by the European Space Agency (ESA), the International Space Science Institute (ISSI), and the national space authorities of its member and cooperating states.
\end{abstract}

Key words. Summer School Alpbach - Space weather missions - Mission concept proposals

\section{Introduction: the objectives of Summer School Alpbach}

Held annually since 1975, the Summer School Alpbach (http:// www.summerschoolalpbach.at) enjoys a long tradition in providing in-depth teaching on aspects of space science and space technology with the aim of advancing the training and working experience of European graduates, postgraduate students, young scientists and engineers.

Participants are given the opportunity to expand and strengthen their knowledge of selected space science and engineering topics in workshops which are part of the Summer School programme.

The purpose of the Summer School is to foster the practical application of knowledge derived from lectures, to develop organisational and teamwork skills and to encourage creativity. Teams compete to design the best project, judged by an independent jury. The teams themselves are responsible for the selection of the subject of the project within the scientific area selected for the year and for the team structure and working methods. Summer School students face great challenges, such as in real life, in a highly compressed form. These include up to 20-hour working days (before proposal submittal), a sudden immersion into new fields and techniques, as well as the difficulties of forming and working together as teams, with scientists listening to engineers and engineers listening to scientists. However, by day 10 of the Summer School, the reward is a proposal for a unique space mission.

The objectives of the Summer School can be summarised as follows:

- motivate participants to seeing space as an exciting and challenging endeavour

- work in international, multi-disciplinary teams by posing challenging topics for designing space missions

- teach a range of scientific topics relevant to possible science missions to design, and teach different aspects of the complex interplay between scientific objectives and requirements, mission and spacecraft design as well as mission costing

- develop the ability to work in teams for a common end, prepare presentations and reports under serious time constraints

- enjoy the unique "Alpbach experience"

Each year, a new topic is offered that is scientifically and technologically challenging for the students. The past 5 years' topics covered the monitoring of natural hazards from space; astrobiology; sample return from the Moon, asteroids and comets; characterising extrasolar planets; monitoring climate change from space.

The Summer School is open each year to 60 selected young science and engineering students and graduates from among ESA member and cooperating states. Students are grouped into four teams, each of which designs a scientific space mission intended to answer some of the key questions relating to the Summer School topic. By the end of the workshop, the teams will have considered not only the instrumentation - having shown that it can meet the scientific objectives and requirements, but also the spacecraft and its orbit: its construction, its sub-systems, its launch and its operations. The cost class for the mission is also estimated. The results of the projects are delivered as short "mission studies" and presented to an expert review panel.

The Summer School Alpbach is a well-tried formula that motivates the young generation to follow space careers and to see space as an exciting and challenging endeavour.

\section{The structure of the Summer School Alpbach}

The Summer School lasts effectively 2 weeks. During the first week, starting on Tuesday (day 1), a ca 16 hours long series of 
lectures is delivered to the students by leading European experts in the topic of the Summer School. The scientific lectures present both the state-of-art of the different aspects of the Summer School topic and the space missions, past, present and planned by the space agencies in the corresponding scientific fields. The lectures cover both general material to introduce the students to the topic and, more specifically, to the more concrete objectives of already existing space missions that address aspects of the Summer School topic. This way, the students are made aware of what is being done and where they should seek for future mission opportunities.

In addition to the scientific lectures, there are also three or more talks about space missions, spacecraft and subsystem design, launchers, orbit selection and in-flight operations. These topics cover both general aspects of space engineering and material relevant to the missions in the year's topic. These topics are usually associated with payload- and instrumentation-specific issues as well as mission/orbit design and operations. It is in these areas that the students need more detailed information for undertaking the design of their selected mission.

Lectures cover days 1 and 2 and the mornings of days 3-5 (day 5 is the Monday of the second week). The bulk of the time, however, is spent by the students organised in teams during the intervals dedicated to the Summer School workshop. The workshop is the most important element of the Summer School Alpbach; through participation in the workshop, students learn how to approach the design of a space mission. Designing such missions involves the students in the definition of mission objectives and its scientific requirements. This leads to designing the spacecraft and payload, and defining the details of the scientific and technical methodology needed to achieve the stated objectives. They come to understand how the general constraints of operations in space, launcher capability and, as a driving constraint, the availability of the required technologies will impact the achievement of these goals. They learn how to form an international team to tackle the many issues connected with space mission design, and how to work together, under pressure, to achieve the goals. These requirements are exactly those that arise in all space missions, and so the Summer School Alpbach workshop is, in a very concentrated way, a good preparation for a career in space science and engineering. In addition, at the end of the mission and architecture definition, an overall cost exercise has to be performed, with the support of provided tools and tutors' expertise, to determine the project class.

The students are pre-allocated into four teams; each team works towards a space mission concept. The students are responsible for organising their team, for selecting spokespersons - two or more - and for assigning tasks among the team so that all areas of the mission concept development are covered. These are very important aspects of the teamwork in the workshops.

The four student teams are each supported by two dedicated tutors. One of the tutors is a scientist, specialised in the topic of the Summer School, while the other is able to offer experience and advice on the engineering aspects of space missions. In addition to these eight tutors, there are up to half a dozen roving tutors, able to provide both specialist science advice and, generally, wide-ranging mission design expertise. A head tutor oversees the workshops and supports the tutors. A daily meeting of the tutors ensures that there is a continuous exchange of information on the teams' progress.

The purpose of the workshop is to develop four mission concepts - one by each team - to the point where a space agency could, in principle, take it over and begin more in-depth feasibility studies. In principle, there should be no duplication of topics among the teams; this is ensured by close liaison among the tutors and of course among the students themselves.

In order to monitor the progress of the project, three semiformal reviews are organised. Each team holds an Objectives and Requirements Review at the end of day 4, a Preliminary Design Review at the end of day 5 and a Final Design Review at the end of day 6. (The weekend between days 4 and 5 is divided between leisure activities and continued work by the students.) These reviews are held separately in each team; lecturers and tutors attend and contribute advice.

The assessment of the teams' work, described in more detail below, is carried out according to specific criteria. These criteria are given to the students at the outset of the Summer School so that they are able to follow their own progress during the workshops.

Defining the mission objectives is obviously the first task of the teams. These objectives are defined in scientific terms, but they also need to be translated to scientific requirements that lead to a definition of the payload/instrumentation and mission/orbit design - together these form the mission architecture. The spacecraft design, the sizing of the subsystems, the budgets for power and telecommunication links follow from the requirements. Mission operations also need to be addressed, at least in outline, to check their conceptual feasibility.

A specific area of concern for all mission proposals is their competitiveness, not only in terms of their scientific objectives but also their overall costs. Although some guidance about cost estimations is available to the teams, a detailed costing, in absolute terms, is beyond the possible scope of the format of the Summer School. However, as this is an important aspect of all training for designing space missions, the students assess a class for their mission, depending mostly on launch mass and the cost of the required launcher. Although this is a relatively crude guide to the overall mission costs, there is clearly a likely major cost difference between proposals, according to whether they require a Vega- Soyuz- or Ariane class of launchers.

A more effective approach to assessing costs in the context of the Summer School is to identify the relationship between mission requirements and the cost drivers of the missions. Teams are explicitly requested to assess the cost drivers of the missions and to identify possible descope scenarios and their impact on the mission objectives.

As the students are invited to be imaginative, the resulting mission concepts are expected to go beyond those currently considered by the space agencies. In many cases, this means that the new mission proposals rely on novel technologies or at least on an extension of current technological capabilities. Students are asked to assess the Technology Readiness Levels and to identify these explicitly in their mission proposal. This exercise, at the same time, leads the students to considering the risks associated with the mission.

The workshop is a unique opportunity for the Alpbach students to explore new objectives and mission concepts in a realistic context and with the support of professional space scientists and space engineers. Teams are therefore encouraged to be as innovative and visionary as possible. They have to justify the scientific objectives and requirements, and they also have to show their understanding of questions related to the technical and programmatic feasibility of the chosen mission. 


\section{Assessing the outcome of the student projects}

At the conclusion of the Summer School, each team prepares and gives a 1-hour presentation on their completed mission concept and submit a written report. The presentations are given to a jury of experts as well as to all the participants of the Summer School. The jury evaluates the mission concepts, based on the presentation and the written mission proposal, according to the four categories of criteria listed below. The assessment of the jury in each of the four categories is communicated to the teams as the final evaluation of their project.

The team projects are evaluated by the jury according to the following criteria:

(A) The scientific case for the mission:

- The overall importance of the mission topic and its scientific objectives

- Statement of scientific requirements to meet the stated objectives

- How much the expected results from the proposed mission advance the field

(B) The technical case for the mission:

- The suitability of the proposed payload as a whole and of the individual instruments to meet the stated goals (matching of payload and instruments to the scientific requirements and mission goals)

- The technical feasibility of the proposed payload, including accommodation and other spacecraft resource requirements such as mass, power and telemetry

- The feasibility of the whole mission concept, including launch and orbit requirements and launcher constraints

- Feasibility of the operational concept and its matching to the mission goals

- Identification of enabling technologies

(C) The competitiveness of the mission:

- How well the mission goals compete with, or complement those of other missions in the field - approved or planned

- How well the mission complements existing and planned research activities in the field in the wide sense

- The value for money of the mission; the quality and breadth of the contribution compared with the expected cost category of the mission

- The identification of descoping options and their impacts on the scientific capability of the mission

(D) Quality of:

- Presentation

- Final report

- Answers to questions

The choice of different topics from year to year provides the basis for the variety, but the imaginative approach by the students to the themes and the way these are translated into mission proposals also contribute largely to the rich spectrum of space missions proposed. Additionally, each topic brings its own challenges, both for determining the science objectives and addressing the different technical drivers for the different topics.

\section{The Summer School Alpbach - 2013 edition}

The topic of the Summer School Alpbach 2013 was space weather. The Summer School addressed innovative mission concepts to study how solar activity can impact near-Earth space, sometimes damaging satellites in Earth orbit and ground-based infrastructures such as electrical power networks or pipelines. The objective of the Summer School was to enable the students to design a space mission that could contribute to a better understanding of the science of space weather and to its more effective monitoring for better forecasting systems. Lectures during the Summer School covered both the scientific knowledge about space weather and its potential effects; in addition, existing space weather forecasting and information systems were presented. Other lectures covered spacecraft design, systems engineering and mission analysis, as well as onboard instrumentation focussed on space weather observations.

The four proposals that were presented by the student teams at the conclusion of the Summer School 2013 are listed here briefly:

CARETAKER (Coronal Analysis Reporting to Earth To Allow Keeping Everything Running)

The mission intends to launch six satellites into an orbit around the Sun at $0.72 \mathrm{AU}$ in order to study large-scale solar eruptions, particularly CMEs. The spacecraft obtain in-situ measurements of the solar wind, as well as observe the corona and the interplanetary environment. Communication is provided $24 / 7$ by two ground stations. The mission provides space weather early warning as well as key scientific data.

PAC2MAN (Photospheric And Chromospheric and Coronal Magnetic field Analyser)

The mission's main goal is to understand and predict the initiation and development of potentially hazardous Coronal Mass Ejections (CMEs) and flares. It also determines the speed and direction of CMEs in order to forecast solar wind conditions near Earth in near-real time. Two spacecraft carry each a payload designed to operate for 6 years. One spacecraft is located at the L1 Lagrangian point of the Sun-Earth system and the other is in a heliocentric orbit, trailing the Earth by $80^{\circ}$.

ADONIS (Atmospheric Drag, Occultation "N" Ionospheric Scintillation)

This mission studies the dynamics of Earth's thermosphere and ionosphere over a full solar cycle, using two spacecraft in low Earth orbit. It investigates satellite drag with in-situ measurements, and ionospheric electron density profiles with radio occultation and scintillation measurements. The low-cost constellation can be 
launched using a single Vega rocket and most of the instruments are already space-proven.

\section{OSCAR (Observatories of Solar Corona and Active Regions).}

This is a mission to identify the $3 \mathrm{D}$ structure of coronal loops, study the trigger mechanism of CMEs in solar active regions and their evolution and propagation processes in the inner heliosphere. It also enables monitoring and forecasting of the CMEs and Corotating Interaction Regions, which are major sources of magnetic storms. OSCAR uses two spacecraft, separated by about $68^{\circ}$ along Earth's orbit, to provide an optimum stereoscopic view of the solar corona. The 5-year mission would launch in 2022-2025.

\section{Dissemination of the Summer School project results}

The proposals prepared by these four teams addressed innovative design ideas of space missions to understand the various aspects of space weather starting from the Sun to the Earth's orbit. The teams presented their mission concepts at the European Space Weather Week 2013. There it was strongly felt that the innovations proposed and experience gained by the participants, if they were published as articles, could serve as material for future Summer School students and would also be useful to many space science students. Since the results and concepts of these proposals were in agreement with the aim and scope of SWSC, the Editors-in-Chief of SWSC invited the teams to submit "educational papers" on their proposal themes.

In order to maintain the consistency and to be fully pedagogic, the above papers were treated like any other scientific paper. In the review process, we based our choice of referees on several usual criteria, which included expertise in space weather and space technology, reputation, our own previous experience of reviewers, etc. However, referees in general wish to see a substantial science part in a paper. But, the above educational papers do not fall into the regular category of science-oriented publications. Each paper however included a good portion of supportive science for solar physics and space weather studies plus a considerable part of innovative instrumentation and technologies required for advanced space-based observations. Therefore, we were compelled to identify referees with the expected expertise and more importantly those who were ready to review as well as support the publication of educational papers. It was rather challenging to identify them (in particular, referees having inclination towards a "science outreach" component). During the review process, firstly due to the missing scientific-result part in these papers, some of the referees faced difficulties in grading the manuscript. Secondly, these proposal-based papers pivoted on challenging design ideas, which demanded high-end sophisticated technology and new development in the instrumentation front and naturally some of the reviewers were influenced to question the conceivability of the projected missions! These also triggered many useful and interesting interactions with some of the reviewers and provided a great experience to handle similar articles in the future. We appreciate all the reviewers for the cooperation extended by them during the review process and the understanding shown by them on the background motivation of these publications. Their constructive comments well shaped the papers. But, the review process took more time than the usual science-article refereeing. We thank all the reviewers for their considerable effort and time at every stage of the review process to keep the standard of these articles.

It was extremely nice to watch the positive attitude shown by the young authors during the review process. For example, the comments from the reviewers were inclined to strengthen the science theme of the papers. Although the authors were less deeply experienced in the wide field of solar physics and space weather, they well utilised each and every aspect of the review reports for their benefits as well as to enhance the scientific base of the papers. It helped to result in a constructive revised version of the paper within one to three iterations. This exercise has certainly led to a useful set of well-documented "educational papers". Additionally, it provided a good platform to encourage and motivate the young students who participated in the Summer School Alpbach to opt for research and/or research-oriented engineering streams for their future careers.

Acknowledgements. The Summer School Alpbach would not be possible without the contributions from the distinguished members of the Alpbach Programme Committee representing the various cooperating space agencies and its outstanding Head Tutor Peter Falkner (ESA) motivating the students by his competence, experience and commitment. Special thanks are also due to the work of Professor Andre Balogh, Distinguished Research Fellow and Emeritus Professor, Imperial College London, who coordinates the lecture contents and the iteration of the lectures every year.

The success and spirit of the Summer School Alpbach originates from its Founder and Father and unique Director, Professor Johannes Ortner. The School itself is now almost the stuff of legend and well recognised and appreciated by the European Space Agency and the space agencies in its member states.

Cite this article as: Gitsch M \& Manoharan PK. The Summer School Alpbach. J. Space Weather Space Clim., 5, E1, 2015, DOI: $10.1051 / \mathrm{swsc} / 2015002$. 